\title{
Genetic and Environmental Effects on Stem Cells and Breast Cancer
}

\author{
Anthony Popkie $\cdot$ Madhuri Kakarala • \\ Carrie Graveel
}

Published online: 11 January 2014

(c) Springer Science+Business Media New York 2014

\begin{abstract}
The remarkable molecular diversity of breast cancers affects therapeutic options and clinical outcome for patients. Understanding the cell of origin and the factors that impact stem cell differentiation and proliferation are crucial aspects for the development of effective breast cancer prevention, diagnostic, and therapeutic strategies. Evidence suggests that molecular subtypes, such as luminal and basal breast cancers, may originate from cells in different stages of epithelial differentiation. Here we review studies that suggest basal breast cancer may have a stem or progenitor cell origin. This work has been augmented by the availability of BRCAl mutant tissues and mouse models of basal breast cancer. We also discuss environmental factors that have been shown to affect luminal breast cancer initiation and progression. Further studies are needed to obtain comprehensive answers to many of these questions, yet the results underscore the importance of breast stem cells in tumorigenesis.
\end{abstract}

Keywords Breast cancer - Stem cells - BRCA1 - HER2/ NEU · Progesterone receptor · BPA · Environmental influences

Madhuri Kakarala and Carrie Graveel have contributed equally to this work.

A. Popkie · M. Kakarala · C. Graveel ( $₫)$

Van Andel Research Institute, 333 Bostwick Ave NE, Grand

Rapids, MI 49503, USA

e-mail: carrie.graveel@vai.org

M. Kakarala

Cancer \& Hematology Centers of Western Michigan, 710

Kenmoor SE, Suite 100, Grand Rapids, MI 49546, USA

\section{Introduction}

Breast cancer is an extremely diverse disease with substantial intertumoral heterogeneity and molecular complexity. Clinically, breast cancers are currently classified on the basis of tumor grade and hormone receptor status, including estrogen receptor (ER), progesterone receptor (PR), and HER2/NEU receptor expression. By using geneexpression profiles we have begun to comprehend breast cancer at the molecular level and currently classify breast cancers into six molecular subtypes: luminal A, luminal B, $\mathrm{HER}^{+}{ }^{+}$, normal-like, basal, and claudin-low. Luminal subtypes commonly express ER and/or PR and have the best clinical outcome. One clinical challenge is identifying and treating luminal breast cancers that do not respond to hormone-based therapy and are driven by other signaling mechanisms. In addition, there are many questions about environmental factors that may promote the development and progression of $\mathrm{ER}^{+}$luminal breast cancers. The basal or triple-negative $\left(\mathrm{ER}^{-}, \mathrm{PR}^{-}, \mathrm{HER} 2^{-}\right.$) and claudin-low subtypes have the worst clinical outcome despite treatment with chemotherapy. Effective targeted therapy for these subtypes has yet to be identified. Currently, breast cancers clinically defined as triple-negative are treated with cytotoxic combination chemotherapy, with platinum-based therapies having the highest response rates.

Results from several studies have implied some breast cancer subtypes have a stem cell origin. In addition to explaining the intertumoral heterogeneity observed among breast cancer subtypes, identifying the cell of origin for each subtype will facilitate the development of effective detection, prevention, and therapeutic strategies. Currently, most breast cancers are treated with cytotoxic chemotherapeutics that target highly proliferating cells. Cancer stem cells comprise a small subpopulation of quiescent cells within the 
tumor. Therefore, cancer stem cells are unaffected by cytotoxic chemotherapeutics and are often driven to a more resistant phenotype by current treatments [1,2]. By distinguishing the cell of origin for each subtype, we will be able to develop successful therapeutic strategies that target the subpopulation of cells that are responsible for tumor progression, resistance, and metastasis. Physiological and developmental factors make breast cancer an ideal model for interrogating the cell of origin. Because the mammary gland primarily develops postnatally and numerous mouse models with altered mammary development exist, we have a profound understanding of the hierarchical organization of the mammary epithelium and the critical points of stem and progenitor cell differentiation. Studies examining normal epithelial differentiation have also identified several distinct markers that can be used to enrich different epithelial cell populations. Epithelial cells that differentiate into luminal cells express "luminal" markers, for example cytokeratins 8/18, ER $\alpha$, and GATA3-binding protein. Basal cells express "basal" markers, for example cytokeratins 5/6, 14, and vimentin. Because luminal breast cancers often express luminal markers and basal breast cancer express basal markers, it has been suggested that luminal breast cancers arise from transformed luminal progenitor cells and basal breast cancers arise from transformed basal progenitors. However, several studies performed in recent years have both clarified the origin of luminal and basal breast cancers and resulted in further confusion. Here we review the involvement of stem cells in the initiation and promotion of several breast cancer subtypes.

\section{Stem Cell Origin in Basal Breast Cancer}

BRCAl is a tumor suppressor gene that is mutated in the germline of approximately $10 \%$ of breast cancer patients. Because most BRCA1 mutant breast cancers are classified as the basal subtype [3, 4], BRCAl mutant tissues have been a valuable model for investigation of the cell of origin of basal breast cancers. The availability of several Brcal mouse models and access to both normal and neoplastic tissue from individuals with $B R C A 1$ mutations have facilitated examination of cancer stem cells within basal breast cancers. Several studies that have used a variety of models and approaches to investigate epithelial differentiation in basal breast cancers have yielded interesting and divergent results. Using cell surface protein markers (CD49f, EpCAM) originally characterized in mouse models [5], Lim et al. [6*•] were able to isolate functional stem, luminal progenitor, and differentiated mammary cells from normal mammary tissue and preneoplastic tissue from BRCA1 heterozygous mutant individuals. Interestingly, an expanded luminal progenitor population was detected among women who carry germline $B R C A 1$ mutations. Comparison of mammary gene signatures with breast cancer subtypes revealed that luminal subtypes were associated with mature luminal signatures, the claudin-low subtype was associated with a MaSC (mammary stem cell)-associated signature, and the basal subtype was associated with a luminal progenitor signature. On the basis of these results, Lim et al. proposed that BRCAl-associated basal breast cancers originate from an aberrant luminal progenitor population. These results seemed to conflict with those of Liu et al., who observed that BRCAl is required for differentiation of $\mathrm{ER}^{-}$stem and/or progenitor cells into $\mathrm{ER}^{+}$luminal cells [7•]. In this study, Liu et al. used a siRNA lentivirus to achieve knockdown of BRCAl in normal human mammary cells. Their results showed that BRCA1 knockdown resulted in an increased population of cells expressing the stem cell marker aldehyde dehydrogenase 1 (ALDH1). ALDH1 was first characterized as a breast cancer stem cell marker by Ginestier et al., by use of the ALDEFLOUR assay [8]. Liu et al. also discovered that histologically normal lobules from women with germline BRCAl mutations were positive for ALDH1 expression but negative for $\mathrm{ER}$, compared with lobules from normal (wild type) BRCAl controls that were $\mathrm{ALDH}^{-}$and $\mathrm{ER}^{+}$. Further examination determined that the $\mathrm{ALDH} 1^{+} / \mathrm{ER}^{-}$lobules had loss of heterozygosity $(\mathrm{LOH})$ for BRCAl compared with adjacent lobules that were $\mathrm{ALDH} 1^{-} / \mathrm{ER}^{+}$and maintained a wildtype BRCAl allele. Together these findings suggest that gene dosage of $B R C A l$ is critical for normal mammary epithelial differentiation. The results from Lim et al. were based on women with BRCAl germline mutations that retained a wildtype $B R C A 1$ allele, whereas the siRNA $B R C A 1$ knockdown in Liu et al. is likely to have resulted in a more substantial $B R C A 1$ knockdown. Therefore, cells that maintain a $B R C A l$ wildtype allele may have altered differentiation that results in luminal progenitor expansion, whereas loss of both functional BRCAl alleles results in altered stem/progenitor cell differentiation and expansion of ALDH $1^{+}$cells. This is supported by evidence of increased ALDH1 expression in mammary epithelial cells with BRCAI LOH. In addition, differentiation of stem and progenitor cells may be affected by accumulating genetic lesions that are maintained by BRCAl deficiency.

A more recent study by Molyneux et al. [9] used mouse models in which Brcal loss of function was directed to either a predominantly basal (K14-Cre) or luminal (BlgCre) epithelial cell. This promoted the origin of cell transformation in a basal or luminal progenitor. Molyneux et al. observed that tumors originating in luminal progenitors histologically resembled human BRCAl tumors and human basal breast cancers. However, tumors originating from the basal cell origin $($ K14-Cre) were 
characterized as metaplastic carcinomas or malignant adenomyoepitheliomas. Molyneux et al. also demonstrated that luminal $\mathrm{ER}^{-}$populations were able to produce multilineage epithelial outgrowths containing myoepithelial, luminal $\mathrm{ER}^{-}$, and luminal $\mathrm{ER}^{+}$cells in a cleared mammary fat pad transplantation assay. The Kuperwasser laboratory performed a complimentary study using disease-free tissue from $B R C A l$ heterozygous mutant and wildtype women [10]. Organoids isolated from these women were lentiviral-infected with a combination of oncogenes and transplanted into humanized fat pads to examine lineage differentiation. Proia et al. observed that luminal progenitors from BRCAl heterozygous mutant tissue had defects in luminal differentiation and developed basal-like differentiation phenotypes.

The discrepancies in these studies may be a result of slight differences in the mouse models (i.e. $p 53$ heterozygous mice vs. $p 53$ floxed mice, different background strains, and different K14-Cre models) and the different cell surface markers (i.e. CD49, CD29, CD24, Sca1 vs. ALDH1) used for cell isolation. It should also be considered that, when isolating cell populations with cell surface markers, the isolates are at best an enrichment of the desired cells and not pure populations. Most importantly, a recent finding by Van Keymeulen et al. [11] suggests that the mammary fat pad transplantation assay, regarded as the standard for determination of cell differentiation potential, may not reflect the differentiation potential of stem cells under normal physiological conditions. Therefore, the use of this assay in determining the differential potential of luminal progenitors into basal cells should be interpreted with caution.

A possible explanation of these different observations is the substantial heterogeneity within the basal and "triplenegative" subtypes. Cluster analysis of 587 triple-negative breast cancers (TNBCs) revealed there are six to seven TNBC subtypes and the subtypes have differential responses to neoadjuvant chemotherapy [12••, 13]. This high level of molecular heterogeneity may be indicative of divergent starting points of transformation along the path of differentiation from an MaSC to a luminal progenitor. In this situation, tumors with a more stem-like, undifferentiated phenotype would arise from a stem cell, whereas other basal-like tumors would arise from a luminal progenitor or a stem/progenitor cell. Proia et al. [10] demonstrated that $B R C A 1$ directly affects mammary epithelial differentiation and that loss of BRCAl alters progenitor cell lineage commitment through regulation of Slug. Therefore, human tissue and mouse models with BRCAl deficiency may represent an altered mammary differentiation profile that is similar to but not identical with that of other sporadic basal breast cancers. Additional studies of basal breast cancers and diverse mouse models are required to clearly elucidate the cell of origin in the diverse subtype of basal breast cancers.

\section{Effects of Environmental Exposures on Breast Stem Cells}

Since genetically inherited breast cancers account for fewer than $10 \%$ of all cases, there is a need to elucidate how environmental exposure promotes expansion of breast stem cells and tumorigenesis. Several epidemiological studies have demonstrated that hormone receptor-positive (luminal) breast cancer risk is increased by lifetime estrogen exposure, including exposure to hormone replacement therapy, oral contraceptives, and nulliparity [14]. Given that luminal breast cancers account for most breast cancers, we must understand the mechanisms by which environmental factors promote luminal breast cancer to improve prevention. For example, diethylstilbestrol, an estrogenic compound given to pregnant women to prevent miscarriages, has been correlated with an increased incidence of breast cancer among women older than 40 years and increased mammary tumorigenesis in adult rodents [15]. Environmental estrogenic compounds also include bisphenol A (BPA), which is found in many plastics and circulating currency, and phthalates, which are present in agricultural fertilizer and pesticides. Exposure of humans to BPA has been shown to induce gene expression changes similar to those caused by estradiol treatment [16]. Genistein, curcumin, and resveratrol are examples of dietary phytoestrogens which can bind to ER and activate estrogenic signaling, albeit with much weaker affinity than endogenous estrogen. Genistein is an isoflavone found in soybeans, curcumin is a polyphenol found in the spice turmeric, and resveratrol is a polyphenol found in grapes and wine. By using endometrial cancer cells and luminal breast cancer cell lines, Gertz et al. [17•] investigated the transcriptional effect of BPA, genistein, and $17 \beta$-estradiol (E2). ChIP-Seq demonstrated that BPA and genistein induced estrogen receptor 1 (ESR1) binding to thousands of sites on the genome. There was significant overlap of genistein and BPA binding with E2 binding sites (genistein $=93.6 \%, \quad \mathrm{BPA}=95.2 \%$ overlap). Quantitative strength of downstream effects was tested by use of ChIPSeq to calculate number of sequence reads in a binding site per million aligned reads (RPM). The signal strength for estradiol, genistein, BPA, and DMSO vehicle control was 21.5, 13.2, 4.1, and 0.15 RPM, respectively. BPA has also been found to induce changes in such breast stem cell regulatory genes as FOXA1 and GATA3, and in CXCL12, a chemokine which can increase metastatic potential [17•]. These studies confirm the effect of BPA and genistein on genome-wide gene expression. 
The impact of BPA dose and route of exposure has been scrutinized both in rodent models and human tissues. BPA has been found in the circulating serum of pregnant women at $0.3-18.9 \mathrm{ng} / \mathrm{mL}$ concentrations [18]. In utero exposure to BPA has been observed to induce early onset of puberty in rodent models [19] and increase the risk of breast cancer [20]. It has been hypothesized that the number of breast stem cells is established in embryogenesis and expanded during thelarche, suggesting that these are particularly vulnerable times during which exposure may have more effect on adult breast cancer risk [1]. Therefore, the timing of exposure to environmental factors during breast development is likely to have a significant effect on breast stem cell proliferation and differentiation. Betancourt et al. [21] examined altered carcinogenesis in mammary glands of rats after prepubertal exposure to BPA and genistein and found that genistein significantly decreased tumor burden whereas BPA treatment significantly increased tumor multiplicity in a DMBA rodent model of carcinogenesis. Proteomic analysis of the signaling pathways demonstrated that BPA induced increases in annexin A2, vascular endothelial growth factor receptor (VEGFR2), and phospho-AKT, whereas genistein reduced levels of all three proteins. These results may explain the different effects of BPA and genistein on cell proliferation and mammary tumorigenesis.

Epidemiologic evidence indicates that soy consumption reduces risk of breast cancer. Reduced risk of breast cancer has been observed in Asian women with high soy consumption in their diets, and this protective effect is diminished in daughters who adopt a diet more characteristic of Caucasian women that is low in soybeans [22]. Currently, the mechanism by which soybeans provide protection against breast cancer is poorly understood. Genistein has been observed to alter DNA methylation throughout the mouse genome and to negate the effect of BPA exposure of DNA hypomethylation [23, 24]. It is reasonable to speculate that genistein may provide protection against breast cancer because of epigenetic effects established during childhood; however, it is not clear which genistein target genes are involved in breast cancer prevention.

The hypothesis that breast stem cell number is programmed in utero raises the tantalizing question of whether breast cancer risk in offspring may be modulated by environmental exposure during prenatal development. To test this, Soto et al. [25] administered BPA to pregnant mice from day 8 to 18 through subcutaneous pumps at $0.25 \mu \mathrm{g} / \mathrm{kg}$ maternal body weight/day, a dose 1,000 fold lower than concentrations in human plasma. BPA treatment altered the mammary gland tissue in embryos, by way of altered extracellular matrix organization, reduced tenascin $\mathrm{C}$ in the periductal stroma, and altered adipogenesis regulatory signaling. BPA-exposed mice also had an increased number of terminal end buds (TEBs) in the mammary gland, and increased TEB area and density, and ductal extension. Similarly, circulating xenoestrogens including phthalates, parabens, and BPA have been associated with increased mammographic breast density, a risk factor for breast cancer, in adult postmenopausal women [26]. To examine specifically whether in utero exposure to BPA increases adult risk of mammary carcinogenesis, a rat model was used with in utero BPA concentrations ranging from 2.5 to $1,000 \mu \mathrm{g} / \mathrm{kg}$ body weight. Carcinoma in situ was found in $33 \%$ of rats exposed to $250-1,000 \mu \mathrm{g} \mathrm{BPA} /$ $\mathrm{kg}$ /day whereas no unexposed rats had such lesions [27]. Hilakivi-Clarke et al. [28], in a similar design, examined whether there may be a protective benefit of genistein exposure in utero among pregnant dams and found that breast tumorigenesis decreased significantly in the offspring. These studies indicate that prenatal exposure to estrogenic compounds may have a significant effect on stem cells and breast development.

The relationship between adult human breast tissue concentrations of BPA and breast stem cell biology is currently under investigation by using explanted cells from reduction mammoplasty tissue. Whether treatment of adult humans with phytoestrogens reduces risk of hormone receptor-positive breast cancer remains controversial, largely due to variances in the dose and form of administration of these compounds in controlled human clinical trials and the lack of mechanistic biomarkers to demonstrate efficacy. Further investigation of dietary phytoestrogens and their effect on breast stem cell proliferation and differentiation is essential to reducing the rate of breast cancer worldwide.

\section{Conclusion}

For cancer treatments to be effective, it is imperative that we delineate which cancers originate from stems cells and are unlikely to respond to chemotherapeutic agents that target highly proliferating cells. This is particularly important for breast cancer, for which there is a large discrepancy in outcomes for molecular subtypes (i.e. luminal vs. basal). To interrogate the origin of basal breast cancer, several laboratories have taken advantage of normal and neoplastic tissue from BRCAl mutant individuals and mouse models of basal breast cancer. These resources have facilitated numerous studies that demonstrate basal breast cancers emanate from a stem or progenitor cell. Most of these studies suggest that basal breast cancers arise from luminal progenitor cells defective in luminal differentiation, whereas other studies infer that MaSCs are the cell of origin. The discrepancies in these results may be 
because of the significant heterogeneity within the basal breast cancer subtype and the variability in the approaches and models utilized. Overall, it is clear that basal breast cancers originate from a non-differentiated progenitor or stem cell and therapeutic strategies need to be tailored to target these cells.

Luminal breast cancers account for the majority of breast cancers and several epidemiological studies have demonstrated that estrogenic compounds increase breast cancer risk. In particular, environmental exposure to such compounds as BPA and genistein may significantly alter genome-wide gene expression and proteomic signaling at critical points in mammary development. More studies are necessary to understand the effects of dosage and timing during prenatal and postnatal development on the initiation and promotion of luminal breast cancers. The results are expected to have a significant impact on breast cancer prevention.

\section{Compliance with Ethics Guidelines}

Conflict of Interest Anthony Popkie, Madhuri Kakarala and Carrie Graveel have no conflicts of interest to disclose.

Human and Animal Rights and Informed Consent This article does not contain any studies with human or animal subjects performed by any of the authors.

\section{References}

Recently published papers of particular importance have been highlighted as:

- Of importance

•- Of major importance

1. Kakarala M, Wicha MS (2008) Implications of the cancer stemcell hypothesis for breast cancer prevention and therapy. J Clin Oncol 26:2813-2820

2. Li X, Lewis MT, Huang J, Gutierrez C, Osborne CK, Wu MF, Hilsenbeck SG, Pavlick A, Zhang X, Chamness GC, Wong H, Rosen J, Chang JC (2008) Intrinsic resistance of tumorigenic breast cancer cells to chemotherapy. J Natl Cancer Inst 100:672-679

3. Lakhani SR, Reis-Filho JS, Fulford L, Penault-Llorca F, van der Vijver M, Parry S, Bishop T, Benitez J, Rivas C, Bignon YJ, Chang-Claude J, Hamann U, Cornelisse CJ, Devilee P, Beckmann MW, Nestle-Kramling C, Daly PA, Haites N, Varley J, Lalloo F, Evans G, Maugard C, Meijers-Heijboer H, Klijn JG, Olah E, Gusterson BA, Pilotti S, Radice P, Scherneck S, Sobol H, Jacquemier J, Wagner T, Peto J, Stratton MR, McGuffog L, Easton DF (2005) Prediction of BRCA1 status in patients with breast cancer using estrogen receptor and basal phenotype. Clin Cancer Res 11:5175-5180

4. Palacios J, Honrado E, Osorio A, Cazorla A, Sarrio D, Barroso A, Rodriguez S, Cigudosa JC, Diez O, Alonso C, Lerma E, Dopazo J, Rivas C, Benitez J (2005) Phenotypic characterization of BRCA1 and BRCA2 tumors based in a tissue microarray study with 37 immunohistochemical markers. Breast Cancer Res Treat 90:5-14
5. Stingl J, Eirew P, Ricketson I, Shackleton M, Vaillant F, Choi D, Li HI, Eaves CJ (2006) Purification and unique properties of mammary epithelial stem cells. Nature 439:993-997

6. • Lim E, Vaillant F, Wu D, Forrest NC, Pal B, Hart AH, AsselinLabat ML, Gyorki DE, Ward T, Partanen A, Feleppa F, Huschtscha LI, Thorne HJ, Fox SB, Yan M, French JD, Brown MA, Smyth GK, Visvader JE, Lindeman GJ (2009) Aberrant luminal progenitors as the candidate target population for basal tumor development in BRCA1 mutation carriers. Nat Med 15:907-913. This was the first study to demonstrate that breast tissue from BRCA1 mutation carriers have an expanded luminal progenitor population and that gene expression profiles of breast tissue heterozygous for a BRCA1 mutation and basal breast tumors were similar to normal luminal progenitor profiles. These results suggest that an aberrant luminal progenitor population is the cell of origin in BRCA1-associated basal breast cancers.

7. - Liu S, Ginestier C, Charafe-Jauffret E, Foco H, Kleer CG, Merajver SD, Dontu G, Wicha MS (2008) BRCA1 regulates human mammary stem/progenitor cell fate. Proc Natl Acad Sci USA 105:1680-1685. Using both siRNA and a humanized NOD/ SCID mouse model, Liu et al. observed that BRCA1 expression is required for the differentiation of $E R^{-}$stem/progenitor cells to $E R^{+}$luminal cells. Examination of human tissue revealed that LOH for BRCA1 was present in $A L D H 1^{+}$lobules but not in adjacent $A L D H 1^{-}$lobules.

8. Ginestier C, Hur MH, Charafe-Jauffret E, Monville F, Dutcher J, Brown M, Jacquemier J, Viens P, Kleer CG, Liu S, Schott A, Hayes D, Birnbaum D, Wicha MS, Dontu G (2007) ALDH1 is a marker of normal and malignant human mammary stem cells and a predictor of poor clinical outcome. Cell Stem Cell 1:555-567

9. Molyneux G, Geyer FC, Magnay FA, McCarthy A, Kendrick H, Natrajan R, Mackay A, Grigoriadis A, Tutt A, Ashworth A, ReisFilho JS, Smalley MJ (2010) BRCA1 basal-like breast cancers originate from luminal epithelial progenitors and not from basal stem cells. Cell Stem Cell 7:403-417

10. Proia TA, Keller PJ, Gupta PB, Klebba I, Jones AD, Sedic M, Gilmore H, Tung N, Naber SP, Schnitt S, Lander ES, Kuperwasser C (2011) Genetic predisposition directs breast cancer phenotype by dictating progenitor cell fate. Cell Stem Cell 8:149-163

11. Van Keymeulen A, Rocha AS, Ousset M, Beck B, Bouvencourt G, Rock J, Sharma N, Dekoninck S, Blanpain C (2011) Distinct stem cells contribute to mammary gland development and maintenance. Nature 479:189-193

12. • Lehmann BD, Bauer JA, Chen X, Sanders ME, Chakravarthy AB, Shyr Y, Pietenpol JA (2011) Identification of human triplenegative breast cancer subtypes and preclinical models for selection of targeted therapies. J Clin Investig 121:2750-2767. This study examined the molecular heterogeneity of TNBC and identified six TNBC molecular subtypes that may be useful in biomarker selection, drug discovery, and clinical trial design.

13. Masuda H, Baggerly KA, Wang Y, Zhang Y, Gonzalez-Angulo AM, Meric-Bernstam F, Valero V, Lehmann BD, Pietenpol JA, Hortobagyi GN, Symmans WF, Ueno NT (2013) Differential response to neoadjuvant chemotherapy among 7 triple-negative breast cancer molecular subtypes. Clin Cancer Res 19:5533-5540

14. Dontu G, El-Ashry D, Wicha MS (2004) Breast cancer, stem/ progenitor cells and the estrogen receptor. Trends Endocrinol Metab 15:193-197

15. Palmer JR, Wise LA, Hatch EE, Troisi R, Titus-Ernstoff L, Strohsnitter W, Kaufman R, Herbst AL, Noller KL, Hyer M, Hoover RN (2006) Prenatal diethylstilbestrol exposure and risk of breast cancer. Cancer Epidemiol Biomarkers Prev 15:1509-1514

16. Hess-Wilson JK, Boldison J, Weaver KE, Knudsen KE (2006) Xenoestrogen action in breast cancer: impact on ER-dependent transcription and mitogenesis. Breast Cancer Res Treat 96:279-292 
17. - Gertz J, Reddy TE, Varley KE, Garabedian MJ, Myers RM (2012) Genistein and bisphenol A exposure cause estrogen receptor 1 to bind thousands of sites in a cell type-specific manner. Genome Res 22:2153-2162. ChIP-Seq was used to demonstrate that GEN and BPA induce a distinct set of transcriptional responses genome-wide in breast and endometrial cells. This suggests a mechanism by which environmental estrogens can alter endogenous estrogen signaling.

18. Schonfelder G, Wittfoht W, Hopp H, Talsness CE, Paul M, Chahoud I (2002) Parent bisphenol A accumulation in the human maternal-fetal-placental unit. Environ Health Perspect 110:A703-A707

19. Nikaido Y, Danbara N, Tsujita-Kyutoku M, Yuri T, Uehara N, Tsubura A (2005) Effects of prepubertal exposure to xenoestrogen on development of estrogen target organs in female CD-1 mice. In Vivo 19:487-494

20. Weber Lozada K, Keri RA (2011) Bisphenol A increases mammary cancer risk in two distinct mouse models of breast cancer. Biol Reprod 85:490-497

21. Betancourt AM, Wang J, Jenkins S, Mobley J, Russo J, Lamartiniere CA (2012) Altered carcinogenesis and proteome in mammary glands of rats after prepubertal exposures to the hormonally active chemicals bisphenol $\mathrm{A}$ and genistein. J Nutr 142:1382S-1388S

22. Ziegler RG, Hoover RN, Pike MC, Hildesheim A, Nomura AM, West DW, Wu-Williams AH, Kolonel LN, Horn-Ross PL,
Rosenthal JF, Hyer MB (1993) Migration patterns and breast cancer risk in Asian-American women. J Natl Cancer Inst 85:1819-1827

23. Day JK, Bauer AM, DesBordes C, Zhuang Y, Kim BE, Newton LG, Nehra V, Forsee KM, MacDonald RS, Besch-Williford C, Huang TH, Lubahn DB (2002) Genistein alters methylation patterns in mice. J Nutr 132:2419S-2423S

24. Dolinoy DC, Weidman JR, Waterland RA, Jirtle RL (2006) Maternal genistein alters coat color and protects Avy mouse offspring from obesity by modifying the fetal epigenome. Environ Health Perspect 114:567-572

25. Soto AM, Brisken C, Schaeberle C, Sonnenschein C (2013) Does cancer start in the womb? Altered mammary gland development and predisposition to breast cancer due to in utero exposure to endocrine disruptors. J Mammary Gland Biol Neoplasia 18:199-208

26. Sprague BL, Trentham-Dietz A, Hedman CJ, Wang J, Hemming JD, Hampton JM, Buist DS, Aiello Bowles EJ, Sisney GS, Burnside ES (2013) Circulating serum xenoestrogens and mammographic breast density. Breast Cancer Res 15:R45

27. Murray TJ, Maffini MV, Ucci AA, Sonnenschein C, Soto AM (2007) Induction of mammary gland ductal hyperplasias and carcinoma in situ following fetal bisphenol A exposure. Reprod Toxicol 23:383-390

28. Hilakivi-Clarke L, Onojafe I, Raygada M, Cho E, Skaar T, Russo I, Clarke R (1999) Prepubertal exposure to zearalenone or genistein reduces mammary tumorigenesis. Br J Cancer 80:1682-1688 